\title{
Comments on: Model-free model-fitting and predictive distributions
}

\author{
Juan A. Cuesta-Albertos
}

Published online: 5 April 2013

C Sociedad de Estadística e Investigación Operativa 2013

I first heard about model-free techniques when I found a previous version of this paper around 2010. The procedure that the author proposed was quite flexible, innovative and nice. Model-free methodology takes advantage of all possible statistical techniques to obtain predictive intervals or sets. The only (and important) difference is that, while the usual procedures pay special attention to the estimation of an underlying model, in the model-free setting this estimation is only an intermediate step in the obtention of a suitable family of i.i.d. r.v.'s which is later used in the predictive step. The fact that it is not required to estimate the model with as much precision as possible allows the user a supplementary freedom to achieve the real goal, which is to obtain a predictive interval as accurate as possible. This fact is made apparent in many points in the paper.

To be honest, in my first reading of the paper I had the feeling that the ideas included in it belonged to some kind of philosophic world because, apparently, there were some points whose justification was based on ad hoc procedures and/or weak heuristic reasonings.

Later, I have had the opportunity to discuss several times model-free techniques with the author and I have become more convinced of their usefulness, and that the theoretical foundations of the procedure are reasonably sound.

Of course, some theoretical points remain to be solved, but those problems also remain unsolved in the usual setting. Therefore, they are of independent interest. In fact, it could be considered as an additional merit of the paper to bring them to the attention of the readers. To mention a couple of them, the procedure proposed in Sect. 2.3 involves the following problems:

This comment refers to the invited paper available at doi:10.1007/s11749-013-0317-7.

J.A. Cuesta-Albertos $(\varangle)$

Facultad de Ciencias, Universidad de Cantabria, Avda. los Castros s.n., 39005 Santander, Spain

e-mail: juan.cuesta@unican.es 
1. Given a space $\mathbf{B}$, a probability distribution, $\mathbb{P}$, defined on $\mathbf{B}^{n}$, and a pseudo-metric $d$ on the set of the probability distributions defined on $\mathbf{B}^{n}$, compute the probability distribution on $\mathbf{B}^{n}$ with i.i.d. marginals closest to $\mathbb{P}$ according to $d$.

As far as I know, this problem is unsolved, even if $\mathbf{B}=\mathbb{R}$, but its interest is big.

2. A similar situation appears when the author proposes to look for the multidimensional Normal which is closest to a given distribution.

This problem is solved for some metrics in the one-dimensional case but I am not aware of the existence of a solution in the multidimensional setting.

I would like to end by mentioning an aspect in the paper in which the point of view of the author and mine differ. It refers to a practical application of the methodology and, consequently, it does not affect the core of the method. I already know the author's answer to my reflection, but I consider that the reader could benefit to know it in order to make up his own mind. The issue is the following:

As stated, a main point of the model-free methodology is to obtain a vector with i.i.d. components, with the appropriate marginal distribution. This distribution is, usually, unknown. However, when using the $\mathrm{MF}^{2}$ methodology, the involved distribution is uniform on $(0,1)$ (see Sect. 4.2) because the author applies an estimation of the conditional distribution function to each observation. Obviously, an estimation of the true function cannot produce a sample of exactly i.i.d. U(0,1)-distributed r.v.'s, but only approximately i.i.d. and approximately $\mathrm{U}(0,1)$ r.v.'s.

My point is that the goal of the model-free methodology could be better achieved if the practitioner, directly, chooses a simple random sample of uniform r.v.'s and then applies the following steps of the proposed methodology. At first sight, as stated in Remark 4.5, it seems that this would allow to get rid of the problems mentioned in this remark.

I want to end my comments congratulating the editors of TEST for the invitation to Prof. Politis to write this nice work which should popularize the model-free methodology in the statistical community. 